BULLETIN OF THE

AMERICAN MATHEMATICAL SOCIETY

Volume 78, Number 5, September 1972

\title{
THE FUNDAMENTAL FORM OF A FINITE PURELY INSEPARABLE FIELD EXTENSION
}

\author{
BY MURRAY GERSTENHABER ${ }^{1}$
}

Communicated December 8, 1971

The purpose of this note is to show that to every finite purely inseparable field extension $K / k$ there is associated in a natural way a symmetric cochain $f: K \times \ldots \times K(\gamma$ times $) \rightarrow K$ of $K$ with coefficients in itself which we call the "fundamental form" of $K$. Its degree, $\gamma$, depends on certain structural properties of $K$. The fundamental form is a derivation when considered as a function of any one variable, all others being held fixed. (It is almost always a coboundary when viewed as a function of all variables.) If $K$ is a tensor product of two intermediate fields then its fundamental form is a certain symmetric product of the forms of the intermediate fields. A weak converse is known and a strong one conjectured.

References in this note to Nakai are to [4] and [5], those to Keith are to [3].

1. Definition. Let $A$ be a commutative $k$-algebra, set $Y^{1}(A)=\operatorname{End}_{k} A$ and for every $n>1$ let $Y^{n}(A)=Y^{n}$ be the set of those $n$-cochains $f$ of $A$ with coefficients in itself which are symmetric as functions of all $n$ variables and which have the property that if all but two variables are fixed then $f$ is a two-cocycle when considered as a function of the remaining ones. If $f \in Y^{n}$, then the $n+1$-cochain $\Delta f$ defined by $\Delta f\left(a_{1}, \ldots, a_{n+1}\right)=$ $a_{n} f\left(a_{1}, \ldots, a_{n-1}, a_{n+1}\right)-f\left(a_{1}, \ldots, a_{n-1}, a_{n} a_{n+1}\right)+a_{n+1} f\left(a_{1}, \ldots, a_{n}\right)$ is in $Y^{n+1}$. This defines the "Nakai operator" $\Delta: Y^{n} \rightarrow Y^{n+1}$. It is easy to verify that for odd $n, \Delta$ is identical with the Hochschild coboundary operator $\delta$ restricted to $Y^{n}$. However, in general, $\Delta^{2} \neq 0$ and the $Y^{i}$ do not form a complex. Those elements of $Y^{1}$ which are annihilated by $\Delta^{i}$ are called "ith order derivations" or simply " $i$-derivations" and form an $A$-module denoted by $\mathscr{D}^{i}$. A 1 -derivation is an ordinary derivation of $A$ into itself. If $A$ is unital, which we henceforth assume, then we denote by $Y_{0}^{n}$ the submodule of $Y^{n}$ consisting of those cochains in $Y^{n}$ which vanish when any variable equals 1 . We then have $\Delta Y_{0}^{n} \subset Y_{0}^{n+1}$, and $\mathscr{D}^{i} \subset Y_{0}^{1}$ for all $i$. If $\varphi \in \mathscr{D}^{i}, \psi \in \mathscr{D}^{j}$ then their composite $\varphi \psi$ is in $\mathscr{D}^{i+j}$ (Nakai). The space $\bigcup_{i=1}^{\infty} \mathscr{D}^{i}$ of all "high order derivations" is thus a ring with an increasing filtration. When $A$ is a finite purely inseparable field extension

AMS 1970 subject classifications. Primary 12F15; Secondary $18 \mathrm{H} 15$.

1 The author gratefully acknowledges the support of the NSF through grants NSF-GP20138 and NSF-GP-29268 with the University of Pennsylvania.

Copyright (C) American Mathematical Society 1972 
$K$ of $k$ Nakai has shown that $U \mathscr{D}^{i}=Y_{0}^{1}$, so for some integer $\gamma$ one has $\mathscr{D}^{1} \subset \mathscr{D}^{2} \subset \ldots \subset \mathscr{D}^{\gamma}=\mathscr{D}^{\gamma+1}=\ldots=\left(\operatorname{End}_{k} K\right)_{0}$. In this case we also have that $\varphi \in \mathscr{D}^{i}$ if and only if $\delta \varphi \in \mathscr{D}^{1} \cup \mathscr{D}^{i-1}+\mathscr{D}^{2} \cup \mathscr{D}^{i-2}+\cdots$ $+\mathscr{D}^{i-1} \cup \mathscr{D}^{1}$, a result due to Keith. This gives an alternative inductive definition of "i-derivations" which is meaningful for not-necessarilycommutative rings but which possibly differs from Nakai's for commutative rings other than purely inseparable field extensions. If $\varphi \in \mathscr{D}^{i}(K / k)$, then it follows from the foregoing that $\Delta^{i-1} \varphi \in \mathscr{D}^{1} \cup \mathscr{D}^{1} \cup \cdots \cup \mathscr{D}^{1}$ ( $i$ times); in particular, $\Delta^{i-1} \varphi$ is a derivation as a function of any single variable. To these results we here add the following:

THEOREM. If $K / k$ is a finite purely inseparable field extension, and if $\gamma$ is the least integer such that $\mathscr{D}^{\gamma}=\left(\operatorname{End}_{k} K\right)_{0}$, then $\operatorname{dim}_{K}\left(\mathscr{D}^{\gamma} / \mathscr{D}^{\gamma-1}\right)=1$.

It will follow that $\Delta^{\gamma-1} \mathscr{D}^{\gamma}$ is a one-dimensional $K$-space any generator of which will be called the "fundamental form" of $K / k$.

2. Proof of the theorem. An approximate automorphism of order $m$ ("higher derivation" in the terminology of Jacobson [2]) of a notnecessarily-commutative $k$-algebra $A$ is a formal polynomial $\Phi_{t}=$ $1+t \varphi_{1}+\cdots+t^{m} \varphi_{m}$ with $\varphi_{i} \in \operatorname{End}_{k} A\left(1=\mathrm{id}^{A}\right)$ such that

$$
\Phi_{t}(a b)=\Phi_{t} a \cdot \Phi_{t} b \bmod t^{m+1}
$$

for all $a, b \in A$. That is, $\Phi_{t}$ is an automorphism of $A[t] / t^{m+1}$ (cf. [1]). This is equivalent to having

$$
\delta \varphi_{i}=\varphi_{i} \cup \varphi_{i-1}+\varphi_{2} \cup \varphi_{i-2}+\cdots+\varphi_{i-1} \cup \varphi_{1}, \quad i=1, \cdots, m .
$$

It follows that $\varphi_{i}$ is an $i$-derivation under the inductive definition valid for noncommutative rings. Those $i$-derivations which appear in approximate automorphisms will be called "special". If $\tilde{k}$ is an extension of the field $k$ and $\tilde{A}=\tilde{k} \otimes_{k} A$ then $\mathscr{D}^{i}(\tilde{A})=\tilde{k} \otimes_{k} \mathscr{D}^{i}(A)$, but the analogous assertion is meaningless for special $i$-derivations since the latter in general do not even form an additive group.

Suppose now that $k$ has characteristic $p>0$ and that $A=k[x] /\left(x^{q}-\alpha\right)$ where $q=p^{e}$ for some $e>0$ and $\alpha$ is some element of $k$. Then $A$ has an approximate automorphism $\Phi_{t}$ of order $q-1$ which is completely defined by setting $\Phi_{t} x=x+t$. This implies that

$$
\Phi_{t} x^{m}=x^{m}+\left(\begin{array}{c}
m \\
1
\end{array}\right) x^{m-1} t+\left(\begin{array}{c}
m \\
2
\end{array}\right) x^{m-2} t^{2}+\cdots+t^{m},
$$

so writing $\Phi_{t}=1+t \varphi_{1}+\cdots+t^{q-1} \varphi_{q-1}$, it follows that $\varphi_{i}$ is an $i$-derivation sending $x^{m}$ to $\left(\begin{array}{c}m \\ i\end{array}\right) x^{m-i}$ for all $m \geqq 0$. It is convenient to denote this $i$-derivation formally by $D^{i} / i$ !, where $D=d / d x$. If we include the case $i=0$, then $\operatorname{End}_{k} A$ can be shown to be a free $A$-module having the $D^{i} / i !, i=0,1, \cdots, q-1$, as a basis. Since $\left(\Phi_{t}\right)^{p} x=x+p t=x$, one 
has $\left(D^{i} / i !\right)^{p}=0$ for all $i>0$. (Writing $i=i_{0}+i_{1} p+i_{2} p^{2}+\cdots+i_{s} p^{s}$ with $0 \leqq i_{0}, i_{1}, \cdots, i_{s} \leqq p-1$, one has

$$
D^{i} / i !=c D^{i_{0}}\left(D^{p} / p !\right)^{i_{1}}\left(D^{p^{2}} / p^{2} !\right)^{i_{2}} \cdots\left(D^{p^{s}} / p^{s} !\right)^{i_{s}}
$$

where $c$ is an integer $\not \equiv 0$ mod $p$.) The highest order of any derivation in End $_{k} A$ is therefore $q-1$, which is achieved by $D^{q-1} /(q-1)$ !.

Let $K / k$ be a finite purely inseparable field extension. By Pickert [6] (cf. also Rasala [7]) there is an extension $\tilde{k}$ (in fact there is a minimal finite one) such that writing $\tilde{A}=\tilde{k} \otimes_{k} A$ we have

$$
\tilde{A} \cong \tilde{k}\left[x_{1}\right] / x_{1}^{q_{1}} \otimes \cdots \otimes \tilde{k}\left[x_{r}\right] / x_{r}^{q_{r}}
$$

where $q_{1}=p^{e_{1}}, \ldots, q_{r}=p^{e_{r}}$ for some $e_{1}, \ldots, e_{r}>0$. Denoting the tensor factors of $\tilde{A}$ by $\tilde{A}_{1}, \ldots, \tilde{A}_{r}$, we have $\operatorname{End}_{k} \tilde{A}=\operatorname{End}_{k} \tilde{A}_{1} \otimes \cdots \otimes \operatorname{End}_{k} \tilde{A}_{r}$, from which it follows that $\operatorname{End}_{k} \tilde{A}$ is generated by 1 and the various $D_{l}^{i} / i$ !. $\left(\right.$ Therefore $\bigcup \mathscr{D}^{i}(\tilde{A})=\left(\operatorname{End}_{k} \tilde{A}\right)_{0}=\tilde{k} \otimes\left(\operatorname{End}_{k} K\right)_{0}$, whence $\bigcup \mathscr{D}^{i}(K)$ $=\left(\operatorname{End}_{k} K\right)_{0}$. This concise proof of Nakai's result is due to Keith.) The highest order achieved by any derivation in $\operatorname{End}_{k} \tilde{A}$ is that of

$$
D_{1}^{q_{1}-1} /\left(q_{1}-1\right) ! \otimes \cdots \otimes D_{r}^{q_{r}-1} /\left(q_{r}-1\right) !,
$$

whose order is $\gamma=\left(q_{1}-1\right)+\cdots+\left(q_{r}-1\right)$. Therefore $\tilde{\mathscr{D}}^{\gamma}=\mathscr{D}^{\gamma}(\tilde{A})$ $=\left(\operatorname{End}_{k} \tilde{A}\right)_{0}$, and $\widetilde{D}^{\gamma} / \widetilde{\mathscr{D}}^{\gamma-1}$ is a free $A$-module of rank 1. It follows that $\mathscr{D}^{\gamma}=\mathscr{D}^{\gamma}(K)=\left(\operatorname{End}_{k} K\right)_{0}$ and that $\operatorname{dim}_{K}\left(\mathscr{D}^{\gamma} / \mathscr{D}^{\gamma-1}\right)=1$, as asserted by the theorem.

3. Symmetric cup products, conjectures. If $f$ is a symmetric $m$-cochain and $g$ a symmetric $n$-cochain of the $k$-algebra $A$ with coefficients in itself, then we define the symmetric $m+n$-cochain $f * g$ by setting

$$
(f * g)\left(a_{1}, \ldots, a_{n+m}\right)=(m ! n !)^{-1} \sum f\left(a_{\sigma 1} \cdots a_{\sigma m}\right) g\left(a_{\sigma(m+1)} \cdots a_{\sigma(m+n)}\right)
$$

where the sum is taken over all permutations of $1, \cdots, m+n$. This is meaningful regardless of the characteristic. One can verify that if $A=k[x] /\left(x^{q}-\alpha\right)$ then the fundamental form of $A$ can be defined and equals $D \cup D \cup \cdots \cup D(q-1$ times), and that if we have a tensor product of such algebras, $A_{1}, \ldots, A_{r}$ with fundamental forms $f_{1}, \ldots, f_{r}$, then the fundamental form of $A_{1} \otimes \cdots \otimes A_{r}$ is $f_{1} * \cdots * f_{r}$. (This is always a coboundary if $r>1$.) It follows that if a purely inseparable field extension $K / k$ is of the form $K_{1} \otimes_{k} K_{2}$, and if the fundamental forms of the factors are $f_{1}$ and $f_{2}$, then that of $K$ is $f_{1} * f_{2}$. We conjecture conversely that if the fundamental form factors then $K$ is a tensor product. This has been shown if one puts certain stringent additional conditions on the factors, but the general question is open.

We remark finally that the "exponents" $e_{1}, \ldots, e_{r}$ of $K / k$ can be determined once $\operatorname{dim}_{K} \mathscr{D}^{i}$ is known for $i=1, \ldots, \gamma$, and these in turn depend 
on the Nakai operator $\Delta$. For $\operatorname{dim} \mathscr{D}^{i} / \mathscr{D}^{i-1}=\operatorname{dim} \mathscr{D}^{i}-\operatorname{dim} \mathscr{D}^{i-1}$ is the number of ways of writing $i=i_{1}+\cdots+i_{r}$ with $0 \leqq i_{l} \leqq q_{l}-1$ $\left(=p^{e l}-1\right)$ for $l=1, \ldots, r$. That is, it is the coefficient of $t^{i}$ in

$$
F(t)=\prod_{l=1}^{r} \frac{1-t^{q_{l}}}{1-t} .
$$

Thus, knowing $\Delta$ determines $F(t)$, from which the $q_{l}=p^{e_{l}}$ can be determined.

\section{REFERENCES}

1. M. Gerstenhaber and A. Zaromp, On the Galois theory of purely inseparable field extensions, Bull. Amer. Math. Soc. 76 (1970), 1011-1014. MR 42 \#1806.

2. N. Jacobson, Lectures in abstract algebra. Vol. III: Theory of fields and Galois theory, Van Nostrand, Princeton, N.J., 1964. MR 30 \# 3087.

3. Sandra Z. Keith, High derivations of fields, Dissertation, University of Pennsylvania, Philadelphia, Pa., 1971.

4. Y. Nakai, High order derivations. I, Osaka J. Math. 7 (1970), 1-27. MR 41 \# 8404.

5. Y. Nakai, K. Kosaki and Y. Ishibashi, High order derivations. II, J. Sci. Hiroshima Univ. Ser. A-I Math. 34 (1970), 17-27. MR 42 \# 1807.

6. G. Pickert, Inseparable Körpererweiterungen, Math Z. 52 (1949), 81-136. MR 11, 313.

7. R. Rasala, Inseparable splitting theory, Dissertation, Harvard University, Cambridge, Mass., 1970.

Department of Mathematics, University of Pennsylvania, Philadelphia, PennsylVANIA 19104 